\title{
Integration of Project Management with Hospital Construction in India
}

\author{
Amber Jain, Dr Akanksha Jain \\ jainamber13@gmail.com \\ B6-303, RPS Savana, Sectorn 88, Faridabad, Haryana 121002 \\ C3- 904, SRS Royal Hills, Sector 86, Faridabad, Haryana - 121002
}

\begin{abstract}
Project Management in India has been one of the most under rated aspect of a construction project. Until recently, it has been observed as more of a burden than being a powerful tool to complete the project efficiently and in a timely manner. Some of the major contributors in the above are adhering to multiple formats, extensive record keeping, processing Project Management data into Project Management information, analyzing the outcomes and implementing improvements at site, etc. However, with rapid globalization, Project Management techniques have been adopted in various construction projects across the country and proved to be highly efficient in completing complex projects in a time bound manner with optimum results and desired quality.

The construction and commissioning of complex commercial buildings like Hospitals mandate the implementation of Project Management processes, more so in Indian market scenario. There is a huge scope for improvement in a construction project by following the PM processes from the inception of the project. The author is currently working as the Planning \&Co-ordination Head for the construction of India's largest Medical Campus. An attempt is made by the author to amalgamate Project Management practices with realtime construction and commissioning of a fully air conditioned hospital in India.
\end{abstract}

Keywords: Project Management, Hospital Construction, Project Initiation, Project Planning, Project Execution, Monitoring and Controlling, Closing.

\section{Project Initiation}

1.1 Reason for starting the Project: The compelling reason to initiate the project along with the detailed financial and operational analysis need to be conductedon a broad level and recorded.

1.2 Business Model: It is very important to study in detail and finalize the business model to be followed for the Hospital planning and design. The type of model chosen shall govern a lot of important design aspects such as quality of service, nature of finish, space planning etc.

1.3 Preliminary Project Report: Further, based on the business model selected, a detailed Preliminary Project Report shall need to be prepared by a specialized agency. The report would contain various aspects like land identification, ground survey, traffic analysis, etc. 
1.4 Principal Architect: It is imperative to shortlist and finalize the Principal Architect at the Project Initiation stage. This would add great value to the discussions from here.

1.5 Assign Project Manager: A competent person with relevant background and strong interpersonal and leadership skills should be identified and assigned the role of Project Manager.

1.6 Detailed Project Report (DPR): A DPR is a detailed plan of action and particulars about the proposed project. The project report will be prepared for a plan of action to be undertaken which covers various aspects viz., technical, financial, marketing, management and social.

1.7 Project Funding: Before the initiation of the Project Planning, various funding sources need to be identified. These usually include, but not limited to Banks, NBFCs, Internal Accruals, etc. The DPR plays a very essential role in case the funds have to arranged from outside.

1.8 Initial Timeline: Should be as realistic as possible. The management may take cue from the previous projects executed or some of the existing projects of similar nature in and around the country. This estimate should be as realistic as possible.

1.9 Initial Requirements: Initial requirements on a broader level like number of beds, specialties, section of society targeted, etc. should be detailed and recorded for proper estimation.

1.10Initial Procurements list: The Initial list for procurement of bought-out items. It should mandatorily include the list provided by theMedical team.

1.11Fund Allocation: The fund allocation, if unclear, should be based on the market standards. If needed a small survey may be conducted to analyze the cost.

1.12Identifying the Stakeholders: The initial identification of Stakeholders should be done.

1.13Identifying Initial Risks: The initial risks should be identified. Feedback from all the identified stakeholders is necessary. A Risk Register should be made to record all the risks identified. This can be updated as the project progresses.

1.14Assign initial team: An initial team needs to be assigned to the Project Manager for kick-starting the project planning and various other works.

1.15Standard Operating Procedures (SOPs): It is very essential to lay down the SOPs at the onset of the project. All roles and responsibilities should be defined recorded and communicated. SOPs enable hindrance free work during the project execution and ensure timely resolution of disputes. If such record is already available with the organization, the same shall be used.

\section{Project Planning}

2.1 The most important thing to do is to assemble a Projects team and a Medical team. These two teams along with the Principal Architect, would work in tandem during all the phases of the project planning and execution.

2.2 The next step would require application for the Change of Land Use (CLU) for a Green Field Project and then apply for Consent to Establish (CTE). In case it's a Brown Field project, we may directly apply for the CTE.

2.3 In order to apply for and enjoy the benefits of LEED, GRIHA, etc. accreditation, necessary consultant may be hired for timely action during execution.

2.4 Identify and list down various packages and sub-packages based on the requirement of the project.

2.5 Lay the outline for planning, in detail, for each package,for the following sub divisions -

2.5.1 Scope - Should be planned in as much detail as possible. This should involve the contribution of the entire planning team and subject matter experts. 
2.5.2 Time - Based on Scope, a WBS needs to be created. This would lead to creating of network diagram. The cumulative duration of various activities involved in the longest path would give the time required for the project completion.

2.5.3 Cost - The sum of cost involved with individual activity shall be termed as project cost. The Project Manager has the authority to add contingency cost. Additionally, the senior management may add what we call Management Reserves.

2.5.4 QA/ QC-This includes a) the industry as well as the organization quality standards relating to the end product and b) various process and procedures that need to be followed (including documentation) to ensure that the set quality standards are met.

2.5.5 Communication - This involves the planning of the communication protocol need to be established and adhered to so that all necessary information flow happens to all the concerned individual/ agencies. This would ensure timely action required.

2.5.6 Human Resource - It is imperative to have the right kind and the right number of human resource at the right time. This is possible only through Human Resource management.

2.5.7 - A very important activity which involves identifying the items to be procured and on which phase of the project. These items need to be listed and their factors like lead time, cost, quality, quantity, etc need to be identified and recorded. This would help in timely procurement of these products.

2.5.8 Stakeholder Management-Stakeholders form the backbone of any project. Stakeholders should be properly identified, recorded, interviewed for feedback.

2.6 Plan for the Commissioning and de-snagging of various systems.

2.7 Scope Validation and final handing over - In case of large projects, the Project Manager may need to get the validation done periodically. This ensures that the project is on track and the results being obtained are acceptable.

The Planning Process may be broadly categorized to the following -

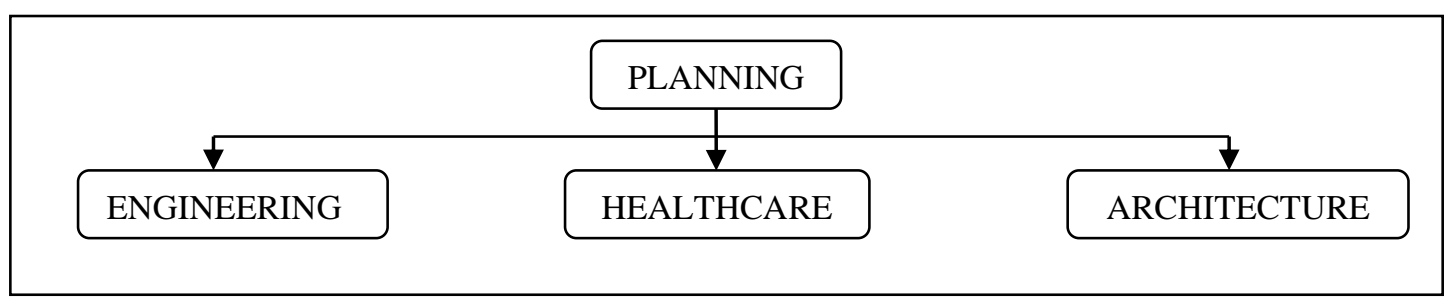

Image 1

\section{Engineering Planning}

The technical part of the planning process involves designing of the building (RCC or Structural), the design of all the services, mainlyair conditioning, plumbing, fire fighting, fire alarm system, access control, lifts, etc. To start the design process, the initial requirements need to be laid down. Parameters like electrical load, air conditioning load, sewage load, domestic water demand, discharge, etc should all be frozen before design finalization.A Design Basis Report (DBR) should be prepared for individual packages. Buffer is added for unforeseen circumstances and a gross value is 
calculated. The calculations are then fine-tuned based on area-wise final requirements. Some broad parameters to keep in mind while finalizing the design of the technical aspect of the Project are-

3.1 Size of the grid while designing of the structure for the optimum utilization of space available.

3.2 Technology and design parameters for all packages (principle, magnitude, components, etc.)

3.3 Input and feedback from end users (doctors) to be taken initially for defining the environment, power requirement, space, etc for various locations.

3.4 Traffic movement inside the building needs to be analyzed and optimized. This includes vertical as well as horizontal travel.

3.5 ALL services drawing need to be analyzed and coordinated for optimum utilization of space. This also helps minimize the floor heights as well as planning efficiently, the partitions.

3.6 Traffic movement outside the main building but within the campus (both initial and final) need to be finalized and frozen for external services, roads and landscape to be designed accordingly.

3.7 Chillers, Libraries and other areas with heavy dead load should be placed as low in the structure as possible.

3.8 The structure should be designed in such a way that it captures most of sunlight during winter and should be least affected by it during summer. A solar path analysis is the best way to achieve that.

3.9 Façade should be kept as simple as possible for seamless integration and should be an integral part of the structure and not a different entity.

3.10In case a Service Floor is constructed, it should be dedicated for Services ONLY

\section{Healthcare Planning}

4.1 Define the number of beds per room for In-patient department that needs to be constructed. Additionally, area for various repetitive locations like IP rooms, OP rooms, corridorsleading to safe and effective delivery of work taking care to avoid crowding (Both OP \& IP) etc., to be frozen.

4.2 Avoiding areas like shelves and damp areas in a room, which could harbor infectious organism.

4.3 Provide natural ventilation and provision for sunlight if possible to aid in sleep wake cycle of patients and to avoid depression in them out of prolonged hospital stay.

4.4 Doctor's time is very important. In order to save on the travel time of doctors from OP to IP and back, the principle of cohorting should be adopted. In cohorting, the OP and IP departments of same/ similar specialty are placed on the same floor.

4.5 Location of Emergency (ER) should be as close to ICU/OT/Radiology as possible. This would help to minimize travel time for Doctor and save critical time for patient in case of an emergency.

4.6 Storage should be planned very meticulously. There should be brainstorming sessions with the operations team for the optimum storage space and the preferred location. The satellite storages should be identified and recorded.

4.7 Location and capacity of pharmacy is very important not only for efficient disbursement of medicine, but also to effectively regulate the crowd.

4.8 Head of Nursing should be consulted for optimum placement of main and satellite Nursing Stations (it should be central in location facilitating monitoring of all patients in the unit from one place). 
4.9 Other facilities required for the efficient working of the Nursing staff and Doctors such as resting room and coffee room would need to be incorporated.

4.10Pneumatic Tube System may be adopted in order to save time and manpower cost. This also improves the efficiency of the system.

4.11 The patient bed movement from one location to another (IP to OT and back, emergency, etc.) should be elaborated, specified and finalized during project planning. The width for the passage, door placement and shall be finalized accordingly.

4.12All provisions for wash/ scrub areas shall need to be discussed in detail and frozen. This is one of the vital inputs for the design of Plumbing works.

4.13The stretcher/ patient lifts should be strategically placed to reduce the lateral movement of patient beds as much as possible.

4.14The location of diagnostics needs to be placed such that it reduces the patient bed movement to the minimum possible.

4.15Medical emergencies like CODE BLUE should be planned for. Placing code blue button close to patient bed easily accessible to Nurse or Doctors and alarm/mobile indication at appropriate place/persons. All details like door width, corridor width, connectivity with OT, ICU, etc should be factored and incorporated. If necessary, a mock drill may be conducted in a mock-up for effective planning.

4.16Pros and Cons of placement of toilet in IP rooms at the entrance or at the back should be thoroughly discussed, analyzed and then finalized. A similar exercise should also be conducted on the size of toilets.

4.17Central AC/Heating in ICUs for meticulous temperature control in order to prevent infections.

\section{Architectural Planning}

5.1 The finishing material used should be medical grade (should be easy to clean and prohibit infection spread)

5.2 The fixtures used should be ergonomically selected based on doctor's requirements.

5.3 Fancy or extravagant features may be avoided unless mandated by the DPR or as desired by the client.

5.4 The façade should be designed to let optimum amount of light to filter in and also provide an excellent view to the people inside. A balance on the amount of area for glazing and solid wall should be established.

5.5 Green cover in the form of Terrace Gardens should be limited and the vegetation may be restricted to green lawns and few shrubs/ short trees. This will keep roots in check and not damage the main structure.

5.6 ALL features which have no direct relation with the operation of the building as a Hospital like suspended planters, etc. should be avoided.

5.7 Wet areas like ponds, fountain, etc should be strictly placed outside the building and should be avoided as much as possible as they call for heavy maintenance.

5.8 The award and subsequent execution of various packages along the timeline of the project should (in generally) follow the logical sequence as stated below(in general, unless there is any different requirement.)

5.8.1 Civil Works (with block work up to lintel level or nil)

5.8.2 Public Health Engineering (PHE) 


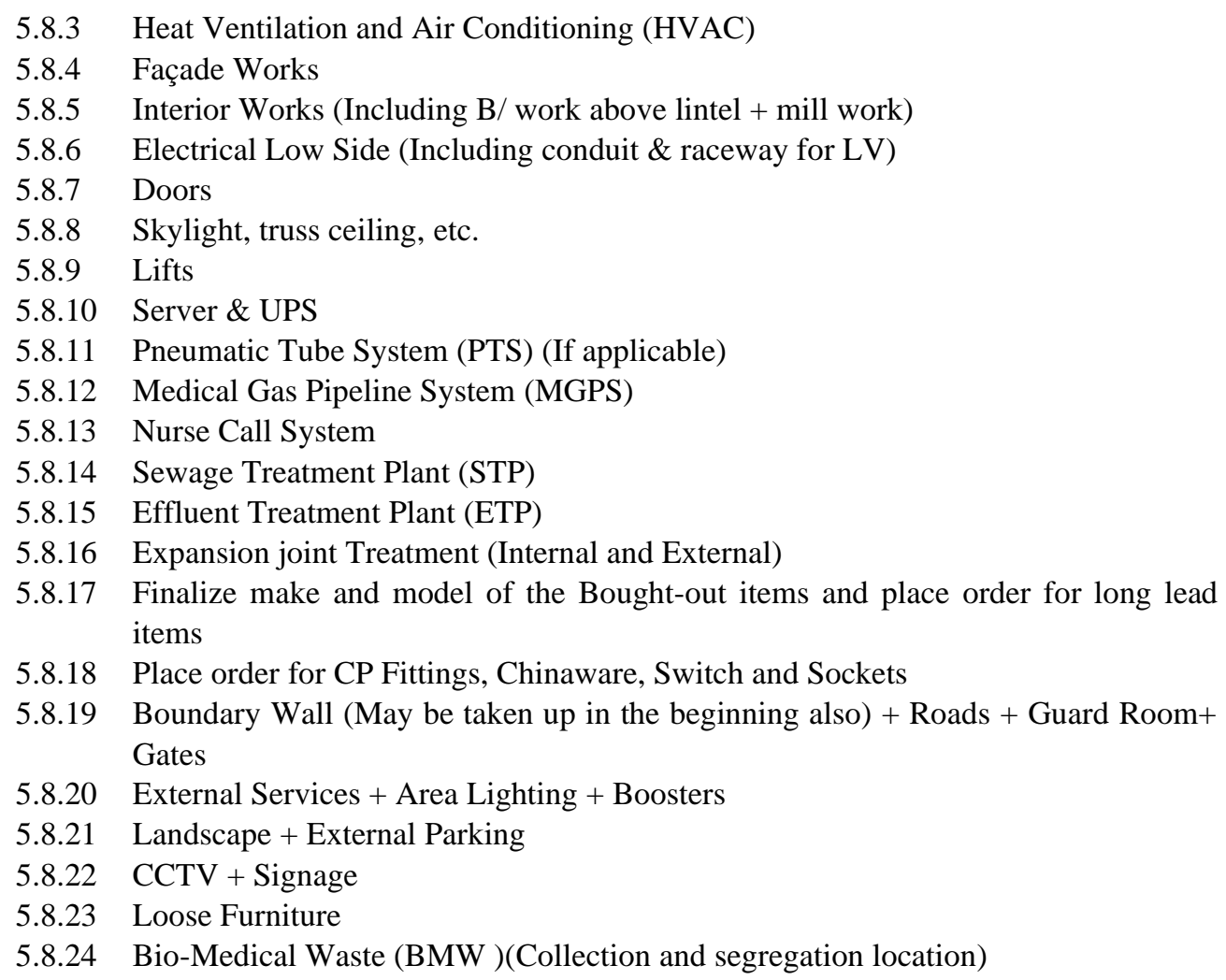

5.9 All High Side packages shall be awarded in parallel with sufficient cushion for charging and commissioning the packages before the project hand over.

5.10Alongside the execution works the following work also need to be taken up-

5.10.1 Power connection from the State Electricity Board needs to applied well in advance and the HT cable need to be laid so as to get electricity in a timely manner.

5.10.2 Domestic Water connection from the local water body + permission to tap Ground Water.

5.10.3 Storm water discharge facility to be identified and necessary provision to be designed and executed.

5.10.4 Temporary nursery may be prepared to nurture saplings at the initial stage and save on cost. It also ensures lesser mortality among plants.

5.10.5 Fire Approvals on the sanctioned drawings. This includes the Fire Tender movement path.

5.10.6 Also necessary is to ensure that the habitable space has minimalistic combustible materials like ply, laminate paneling, rock wool, etc.

5.10.7 Special attention is needed to detail the finishing of Fire Escape Route. It is advisable to line/ layer the route with fire retardants. 


\section{Project Execution}

6.1 A dedicated Planning \& Monitoring and Document Control team needs to be in place at the inception of the project.

6.2 A well experienced Architect and a Structural Designer should be taken on board before the start of the project. For a large project, they would definitely contribute in savings more than their cost.

6.3 Documents need to generated periodically and filed for future and timely reference.

6.4 The work performance data should be converted to work performance information. This information shall help take various key decisions over the tenure of project execution. It also acts as a valuable input for future projects.

6.5 All work schedules should be in coordination with resource mobilization and manpower mobilization plan.

6.6 Work Performance data should be collected to generate a Work Performance Information and communicated to all concerned.

6.7 Intermediate milestones should be strictly monitored and adhered to.

6.8 Site Entry/ Exit locations, earth/ debris dumping location, loading/ unloading areas, location for builder/ passenger hoist, workshops, yards, material storage areas, Offices for vendors, etc. should be identified before starting the work. The movement of man and machinery should be kept fixed at all locations.

6.9 Two agencies for water proofing in case of large water proofing areas. One for toilets water proofing and other for terrace, balconies and other open to sky areas.

6.10Ensure proper treatment and protection of expansion joints. Protection is very important to prevent re-work.

6.11MANDATORILY, PHE shall be awarded first followed by HVAC Low Side. Other packages shall follow.

6.12Façade (glass) works for a floor need to be completed before the starting of the Interior Works for that floor.

6.13All decisions need to be TIME BOUND!

6.14Stores: Safe and secure storage location for bought out items needs to be prepared/ identified. Bio Medical Equipment (BME) requires dust free and climate controlled storage which should be made ready on arrival. The material should be ordered and received depending on the availability of the storage. Or a large storage could be built with a capacity for $30 \%$ of total material. The delivery should be scheduled accordingly.

6.15Large projects should use an efficient MIS in order to record and track the movement of various raw and finished goods arriving in/ for the project.

6.16Although individual housekeeping shall be in vendor's scope, a dedicated housekeeping team should be kept ready to dump the debris collected at site.

6.17Debris removal should be efficient so as to minimize the spread of air pollution inside the building.

6.18All skylights shall need to be closed/ covered timely in order to prevent rain water from entering the structure.

6.19Cold/ Construction joints shall need to be treated properly in order to prevent damage to the interior works. 


\section{Monitoring \& Controlling}

The Monitoring and Controlling process oversees all the tasks and metrics necessary to ensure that the approved and authorized project is within scope, on time, and on budget so that the project proceeds with minimal risk. Monitoring and Controlling process is continuously performed throughout the life of the project.

The Key tasks of the Project Manager during this phase are -

7.1 Schedule Control

7.2 Cost control

7.3 Quality Control

7.4 Scope Verification and Change Control

7.5 Performance Reporting

7.6 Risk Control

7.7 Contract Administration and

7.8 Complete Monitoring and Controlling phase review

The process of Monitoring and Controlling involves contribution from every individual in the team starting from project sponsor to the field staff at various levels.

\section{Testing \& Commissioning}

Although every system is tested in totality while commissioning, it is imperative that every system is tested for defects during the installation phase. Intermittent testing should be included in the scope of the agency awarded with the work. Different types of tests should be defined in the contract and the necessary codes mentioned for clarity .Necessary checklist and other documentation should be prepared and maintained. Systems needing to run for extended duration should be manned and monitored diligently.

8.1 Electrical Low Side:

8.1.1 The $11 \mathrm{kVA}$ substation is checked for faults. Megger values for the entire system are checked for the fault in any cable. The HT panel is then switched ON and the power transmitted to all the MV rooms. The rising main and floor panels are checked for on all floors.

8.1.2 The checking for fault then moves to various loops on each floor which includes checking of lights, power points, etc.

8.1.3 UPS system need to be in place for the entire system to be checked in totality.

8.1.4 Last but not the least to be noted is that all earth pits need to be in place and checked for functionality before switching on the entire system.

8.2 HVAC Low Side:

The testing of HVAC system involves, broadly, following steps -

8.2.1Flushing \& cleaning of condenser pipe, Cooling Tower at least 3 times to ensure proper cleaning.

8.2.2 Chilled water system piping cleaning. We need to ensure that the drainage points for water drainage are made ready. Since the wash is done using Chlorine water, it is imperative that the drainage system should be complete till the STP. 
8.2.3 Dry cleaning of ducts is done parallel wherein all the AHUs are run in full capacity to blow out the dust settled in ducts during the erection phase.

8.3 Drinking/Potable Water:

8.3.1 Water Tank washing: The water tanks are washed in the sequence of their construction, Raw water tank $\rightarrow$ Fire Water tank $\rightarrow$ Treated water.The Hydro pneumatic pump then cleanses the entire system through all vertical shafts. Individual washrooms at every floorare used as drainage points.

8.3.2 Then follows the treated water to $\mathrm{RO} /$ Drinking water (flushing)

8.3.3 Just like in HVAC system, we need to ensure here too that the Drainage water line has been laid till STP and tested.

8.3.4 STP treated water shall be pumped to Terrace water tank. Then connected to flushing system.

8.4 Fire Fighting: This is relatively easy to commission. Apart from pressure testing of various fire zones, this system also needs to be first checked for faulty sprinklers along with trapped air in the system.

8.5 Building Management System (BMS): BMS plays a very critical role in making a building smart. It integrates all the major systems and monitors their functioning to obtain the best possible combination based on the environment. Importance of BMS can be judged from the fact that systems linked to a BMS typically represent $40 \%$ of a building's energy usage; if lighting is included, this number approaches to $70 \%$. Fire alarm systems and elevators should also be linked to a BMS for efficient monitoring. Smart Buildings (ones integrated by BMS) are becoming more and more popular as they reduce energy costs, enhance productivity and building operations and support sustainability efforts. This mandates the need to select efficientBMS software to integrate, monitor and optimize the building systems.

\section{De-Snagging}

A snag list, which is also sometimes referred to as a snagging report, is a list of all the items that need to be completed, fixed, re-done, or addressed before a project is finished. One may have also heard this referred to a punch list.

During this walk-through, each person will point out any items that remain unfinished, any flaws (such as the wrong cabinet fixtures or crooked light switch plates), or repairs (like scuffed floorboards or nails sticking up from floors) that must be done in order to complete the project. Often, the resulting snag list or punch list must be completed in order to receive final payment.

\section{Closing and Handing Over}

The Closing phase includes all the activities necessary for the project office to close the project. Project Close may be signified by system acceptance and transfer to the support organization, or by official system retirement or replacement. It is important that lessons learned during the project are captured and that project information is properly archived. This phase marks the end of the project's operation, including transferring operations and/or data to a follow-on system (as applicable) and retirement of any legacy system. This phase includes archiving project data and documenting final lessons learned.

10.1 Handing Over is not a onetime process but a continuous one. Various areas should be made ready and handed over to the client from time to time based on their requirements. Areas like Labs, 
CSSD, OTs, ICUs, Equipment Rooms, etc require specialized vendors and an early handing over of these areas is necessary for the specific vendor to complete their work on time.

10.2 For common and other areas, the handing over should be complete room by room and floor by floor. Every room should be tested for power points, lights, switch sockets, LAN ports, air conditioning, wet points, hot and cold water functioning, etc and the key handed over to the client.

10.3 All the contracts should be formally closed and completion certificates issued to the various agencies.

\section{PARTY!}

\section{Acknowledgements}

Special thanks to Col (Retd.) Harendra Singh for guiding and providing valuable feedback from his vast experience. Additional thanks to Ar. Khushal Gupta, Mr Hardik Bhate for reviewing the paper and providing their valuable feedback.

\section{References}

1. https://defectspro.com/punch-lists-101/snag-list-vs-punch-list-answers-you-need/

2. https://www.bestpractices.ca.gov/sysacq/projectlifecycle.shtml\#Project_Management 